\title{
Location choice for a continuous simulation of long periods under changing conditions
}
Fabian Märki
David Charypar
IVT ETH Zurich ${ }^{\text {a }}$

\author{
Kay W. Axhausen \\ IVT ETH Zurich
}

\begin{abstract}
The authors propose a location choice procedure that is capable of handling changing conditions of aspects with different time horizons. It integrates expected travel time, current location effectiveness, prospective location effectiveness, and individual unexplained location perception into a decision heuristic that considers different planning horizons simultaneously and decides on-the-fly about future location visits. Multiple simulation runs illustrate agents' location choice behavior in various situations and confirm that the model enables agents to simultaneously consider seasonal effects, weather conditions, expected travel times, and individual unexplained location preference in their location choice.
\end{abstract}

Keywords: location choice; multi-week schedules; continuous activity generation and scheduling; microscopic travel demand simulation; Continuous Target-Based Activity Planning (C-TAP)

\section{Introduction}

Microscopic travel demand simulation software uses a direct representation of virtual people (usually referred to as agents) to generate demand in the form of activity plans. This leads to a high computational complexity that often results in computational performance and memory issues. Microscopic models typically introduce constraints to counter such issues. For instance, Balmer (2007) limited the maximum simulation horizon of standard size scenarios to a single day, making it difficult to investigate effects occurring over a period of days or weeks. Another limitation of Balmer's model is that agents must commit to a specific day plan, making it challenging to simulate unexpected events realistically (Charypar et al. (2009); Dobler et al. (2012)). Including such flexible behavior requires a different simulation approach that is capable of modeling demand continuously over multiple days and weeks, i.e., agents should be able to make decisions about upcoming activities on-the-fly and with an open time horizon (see also empirical insights from the work of Doherty (2005)).

We proposed a microscopic travel demand simulation in Märki et al. (2012a) and Märki et al. (2012b) (we refer to it as CTAP - Continuous Target-Based Activity Planning) that is capable of modeling demand continuously (i.e., continual in time over long periods) by using behavioral targets to guide agents through their decision space. These targets are closely related to observed behavior, e.g., the execution frequency or time spent on a given activity, and can take account of exogenous effects like social and cultural norms. Our agents continuously track their performance and compare it to their behavioral targets using observation windows of different durations. Deviations from the desired behavior cause discomfort, which is conveyed to a planning heuristic that makes decisions about which future activities the agents should execute. This enables agents to react spontaneously to unexpected events. At the same time, it also reduces memory consumption because agents do not need to keep track of complete schedules, making simulation periods of several months feasible.

The aim of this work is to address the question on how to integrate location choice into C-TAP while preserving its ability to simulate long periods in reasonable time and keeping its memory footprint small. The envisaged procedure also needs to be able to consider changing conditions of aspects with different time horizons like long-term phenomena (seasonal effects), midterm aspects (recurrent visits of relatives or changing weather conditions), and short-term elements (expected travel times). We

\footnotetext{
amaerki@ivt.baug.ethz.ch
}

a maerki@ivt.baug.ethz.ch 
propose a combination of expected travel time, current location effectiveness, prospective location effectiveness, and individual unexplained location preference as basis for the continuous location choice procedure. The combination of these elements not only allows investigations of aspects with different time horizons but also enables variety seeking and produces realistic location choice decisions.

The remainder of this paper is structured as follows: first, we will introduce the target-based approach and discuss the decision heuristic used by the model. The section thereafter will start with a comparison of continuous location choice (i.e., continual in time considering changing conditions of aspects with different time horizons) to location choice used in single-day simulation models. This will be followed by an outline of the continuous location choice procedure, its components, and their relevance in the decision process. The subsequent section will validate the proposed continuous location choice procedure by considering seasonal aspects, weather conditions, location accessibility, and individual perception for leisure activities. We will conclude the paper by summarizing the highlights of the proposed approach and providing an outlook on future research.

\section{Related Work}

The target-based approach shows similarities to the need-based theory introduced by Arentze and Timmermans (2006, 2009). Whereas Arentze and Timmermans postulated that needs motivate people to execute activities, we assume that people form a conception of their desired performance as they reported in the data collected by the Swiss Federal Statistical Office (BFS) (2006) as well as in other travel diaries (e.g., Axhausen et al. 2007, 2002; Schönfelder 2006). Peoples' conception of performance is transformed into targets. Consequently, targets are directly observable in the available data, and it is therefore possible to overcome the hidden source problem of the need-based model. Nonetheless, we kept C-TAP generic in the sense that the satisfaction of needs may still be one possible target in C-TAP. We incorporate Winston's (1982) suggestion to use time-dependent utilities for activities (see also Axhausen (1990, 34-38) for a summary or Gliebe and Kim (2010) for recent work in this tradition) and introduce timedependent effectiveness functions to describe the effectiveness of activities and locations with respect to discomfort reduction. Since different locations can provide different effectiveness definitions, location effectiveness becomes an important element of the location choice procedure. The individual unexplained preference for a certain location is modeled as a random variable similar to the proposal of Horni et al. (2012). The target-based model was introduced in Märki et al. (2012a) and validated in Märki et al. (2012b) using an existing six-week continuous travel diary (Axhausen et al. (2007); Schönfelder (2006)).

\section{Target-Based Model}

This section introduces a microscopic travel demand simulation that enables a continuous simulation of several weeks. Agents, representing virtual people, are the central component of C-TAP. Each agent is motivated to execute activities, specifying his or her desired performance beforehand through behavioral targets. Targets describe agents' internal conditions based on personal desires, preferences, and goals. Deviations from these behavioral targets result in discomfort, which induces agents to take action against the deviation; higher deviations result in greater discomfort, which in turn leads to a greater urge to take action. Agents can reduce their discomfort by executing activities at different locations, and we assume that agents prefer activity/location pairs that provide more discomfort reduction. This is similar to the work of Arentze and Timmermans (2009), who proposed that activity utility is a function of need reduction. The model uses effectiveness functions to specify the efficacy of discomfort reduction at a specific location, i.e., effectiveness functions describe the external conditions agents experience when they interact with their environment.

\subsection{Targets}

The core assumption of this work is that people are motivated to execute activities and that they have a conception of their motivation in terms of the performance they want to achieve. People specify this performance through behavioral targets and try to comply with them across observation windows of different durations. For instance, a person might wish to exercise for roughly two hours twice a week. This person might be satisfied with one hour of exercise once a week and state that up to 2.5 hours of 
exercise three times a week would still be compatible with his of her weekly schedule. The targeted behavior could be transformed into targets as follows:

- The percentage of time target defines the amount of the time a person would like to spend on an activity within an observation window. In order to simplify the modeler's task, it is possible to specify the total execution duration, which is then internally converted to the percentage of time target. For the above example, the modeler would specify a target value of two hours of exercise, a bandwidth of ${ }_{-1}^{+0.5}$ hours (the upper and lower bounds of the target value) and an observation window of one week (see Fig. 1a).

- The frequency target defines the number of activity executions a person would like to accomplish within an observation window. For the above example, the modeler would specify a target value of two executions with a bandwidth of ${ }_{-1}^{+1}$ executions and an observation window of one week.

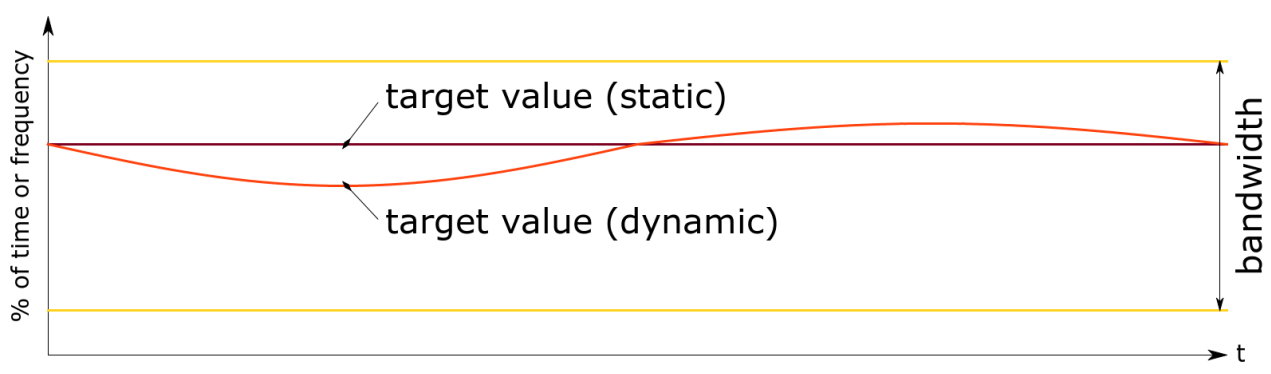

(a) Schematic illustration of a target configuration that defines the average time a person would like to spend on executing an activity. Target values as well as upper and lower bounds (which define the behavioral bandwidth) can be static or dynamic and are therefore modeled as functions in time.

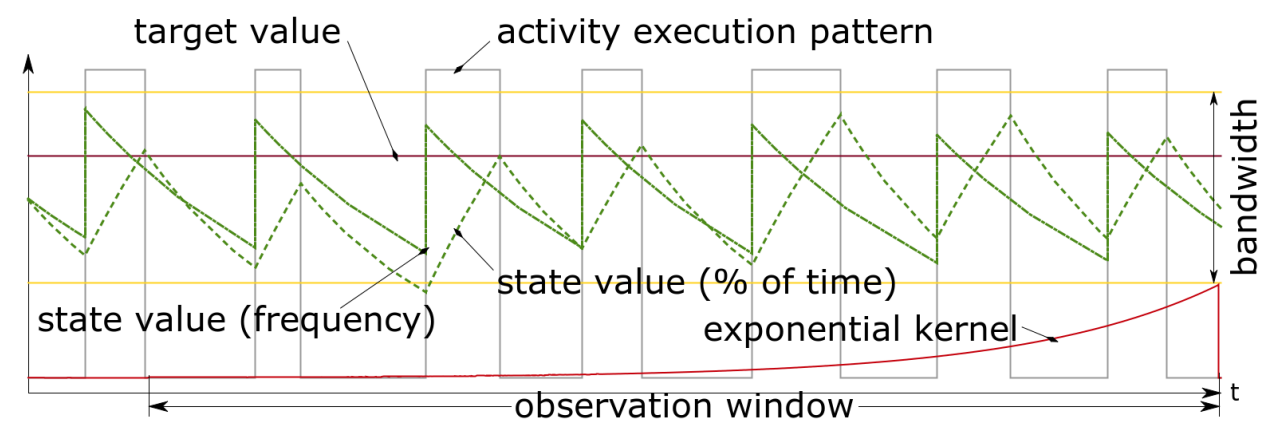

(b) Schematic illustration of performance monitoring. The state value (dashed green line) is calculated through a convolution of the activity execution pattern with an exponential kernel, resulting in an exponentially weighted moving average. The observation window, in which the person tries to comply with the target, defines the kernel length.

Figure 1: Illustration of agent configuration and performance monitoring.

Playing tennis, swimming, and hiking are all forms of exercise. At the same time, playing tennis is also a chance to socialize or even a means of maintaining business relationships (see, e.g., Arentze and Timmermans (2009) for a discussion of multipurpose activities). C-TAP allows for activities to serve multiple targets, and it is possible to assign a target to several activities. This facilitates the configuration of interacting effects, as outlined above.

The presented model also supports static and dynamic targets. Static targets are used to model constant behavioral patterns and dynamic targets to model variable behavioral patterns (also referred to as behavioral rhythms). Behavioral rhythms are conditions in which activity executions vary across observation windows (e.g., leisure activities might be more common during weekends than weekdays). 
Agents monitor their performance during the simulation and compare state values to their target values (see Fig. 1b). State values are exponentially discounted over the observation window of targets. This simulates a forgetting process in which agents give more weight to recent behavior and gradually discount their past performance.

\subsection{Effectiveness Functions}

People seem to have time-dependent preferences for executing activities and/or visiting locations. Reasons for this behavior can be manifold, and they vary according to constraints (e.g., opening hours), norms (e.g., business hours), and contingencies (e.g., weather conditions) as well as combinations of such effects. Effectiveness functions are a simple but comprehensive way to describe such interdependencies and are expressed as the percentage of execution effectiveness. Effectiveness functions inform agents about the efficacy of activities and locations with respect to discomfort reduction. This is similar to the work of Winston (1982), who proposed time-dependent utilities for activities (see also Axhausen (1990, 34-38) for a summary or Gliebe and Kim (2010) for recent work in this tradition). Possible effects that can be modeled by effectiveness functions include:

- Shop opening hours for daily shopping activities. Agents can use this information either to determine whether they can execute a shopping activity without delay and for how long, or to estimate how long it will take until the next opportunity to engage in that activity will arise. Since effectiveness functions can be location dependent, it is also possible to model location-dependent shop opening hours. Furthermore, the effectiveness function can also contain time-dependent information about shop crowdedness. We assume that shopping in overcrowded shops is less efficient (smaller value) and therefore takes longer.

- Circadian rhythm for sleep activities. This function specifies the light intensity. Agents can, for instance, use this information as an indication of sleep effectiveness. We assume that people sleep at night and have already adapted to their current time zone.

- Business hours for work activities. This function can be seen both as a cultural norm (different cultures may keep different business hours) and a social norm (social groups, e.g., professions, may have different business hours). Agents can use this information as an indication of work effectiveness. We assume that people depend on coworkers to be able to do their work (the degree of dependence can vary according to the profession).

\section{Planning Heuristic}

The aim of the planning heuristic is to schedule activities based on agents' preferences and goals (defined by targets) as well as external conditions (defined by effectiveness functions) agents experience when they interact with their environment. Existing approaches to agent-based microsimulations have exhibited various disadvantages when it came to activity scheduling. Balmer (2007) re-planned the same day until he produced an optimal state. This procedure led to high computational costs. Kuhnimhof and Gringmuth (2009) struggled with inflexibilities when agents should have spontaneously reacted to unexpected events. Charypar and Nagel (2006) formulated the planning procedure as a reinforcement learning problem and reported that this approach performed poorly for large scenarios. This work uses a planning heuristic to overcome the above limitations.

The proposed heuristic applies a decision scheme that uses a continuous planning approach, i.e., agents plan upcoming activities on-the-fly considering different planning horizons. Because agents make decisions as they go along, they can react spontaneously to unexpected events and continuously adjust their decisions. As a result, the proposed decision scheme supports within-day replanning by default. The proposed decision scheme also implements a forgetting process in which agents give more weight to recent behavior and gradually discount their past performance (see Fig. 1b). Similarly, agents also pay more attention to their near future and less to opportunities further in their future (see Section 4.2.4 for a description). Applying such a decision scheme limits agents' view into their past and future. This has similarities to reality, where people cannot include their complete record and every prospective opportunity into their decision-making process. Because agents only consider a locally limited time period for their decisions, it also helps to overcome computational demand and memory issues. Since a heuristic is intended to approximate a good solution, it is also possible to use incomplete knowledge about the state of mind and plans of other agents. This is also helpful since complete knowledge generally leads to high computational and memory costs, especially if simulations are executed 
on distributed computation environments in order to speed up the simulation. One could argue that people seek optimal day plans and that applying a heuristic makes this infeasible. However, other authors (e.g., Schlich 2004; Simon 1955) doubt that behavior can be explained as a function of utility maximization that aims at a global optimum. People seem to maximize their utility, but with a limited view similar to a search for a local optimum.

The next subsection will outline the decision procedure, which will be followed by a detailed explanation of its components.

\subsection{The Decision Procedure}

An agent decides upon his or her next action by applying a three-step decision procedure to all his or her promising activity/location pairs. This decision procedure combines different planning aspects into a heuristic function. These aspects include a comparison of the past performance with the targeted performance, an evaluation of the immediate execution options, an estimate of the prospective activity execution opportunities, a consideration of the travel time, and a random term modeling individual perception. The heuristic function $H F$ is defined as:

$$
\begin{aligned}
& H F\left(t_{t s}, t_{e s}, t_{e e}\right)=\underbrace{D R\left(t_{e s}, t_{e e}\right) \cdot E E\left(t_{e s}, t_{e e}\right) \cdot L A\left(t_{e e}\right)} \cdot \operatorname{ETR}\left(t_{t s}, t_{e s}, t_{e e}\right) \cdot(1+\epsilon) \\
& \text { step 1: activity duration } \\
& \text { step 2: gain per time invested } \\
& \text { step 3: random fluctuation [optional] }
\end{aligned}
$$

where $D R$ is the discomfort reduction, $E E$ is the execution effectiveness, $L A$ is the look-ahead index, $E T R$ is the execution time ratio, $(1+\epsilon)$ is a scaled random term, $t_{t s}$ is the start of travel, $t_{e s}$ is the start of execution, and $t_{e e}$ is the end of execution (see Section 4.2 for an explanation of the individual factors).

In the first step, an agent determines the optimal end of execution $t_{e e}$ by maximizing the product of $D R \cdot E E \cdot L A$ using a numerical optimization known as Brent's method (Press et al. 2007, 496-499). This step defines the duration of the activity and thus, the presented model generates activity durations as a function of agents' state (see also Section 7.1). The integration of $D R$ results in a preference for alternatives (activity/location pairs) which provide discomfort reduction, using $E E$ favors alternatives which are commonly selected (e.g., based on cultural or social norms), and the inclusion of $L A$ fosters the selection of alternatives that have a certain inflexibility with regard to being postponed to a later point in time. In the second step, the agent multiplies the maximized value by $E T R$, introducing a preference for accessible locations. So far, the decision procedure is homogeneous for all agents in the sense that agents having the same state and facing the same situation will make the same decision. Clearly, this does not match the heterogeneity of observed behavior (see, e.g., Chikaraishi et al. 2010; Schönfelder 2006). In order to introduce variation, agents can incorporate a scaled random term into the decision-making procedure as an optional third step. In the final step, the agent decides to implement the activity/location pair yielding the highest value of the heuristic. This is similar to the work of Arentze and Timmermans (2009), who proposed that activity utility is a function of need reduction and implemented a procedure that selected activities in respect thereof.

\subsection{Components of the Planning Heuristic}

\subsubsection{Discomfort}

Discomfort builds on targets and is a function of the difference between targeted and actual behavior (i.e., the difference between target values and state values) and thus, it is a measure that considers agents' past performance. Discomfort levels identify the 
urgency an agent experiences to take action against the origin of the discomfort. The discomfort an agent feels from an activity at time $t$ is defined as:

$$
\begin{gathered}
D(t)=\sum_{k=1}^{n}\left(f_{\text {target }}^{k}(t)-f_{\text {state }}^{k}(t)\right)^{2} \cdot\left\{\begin{array}{cc}
w_{1}^{k} & \text { if } f_{\text {state }}^{k}(t) \leq f_{\text {target }}^{k}(t) \\
w_{2}^{k} & \text { otherwise }
\end{array}\right. \\
w_{1}^{k}=\frac{1}{\left(f_{\text {target }}^{k}(t)-f_{\text {lower-bound }}^{k}(t)\right)^{2}} \\
w_{2}^{k}=\frac{1}{\left(f_{\text {target }}^{k}(t)-f_{\text {upper-bound }}^{k}(t)\right)^{2}}
\end{gathered}
$$

where $k$ is the index of the target, $f_{\text {target }}^{k}(t)$ is the function defining the target value, $f_{\text {state }}^{k}(t)$ is the function used to calculate the agent's state value, $f_{\text {lower-bound }}^{k}(t)$ is the function defining the lower bound, and $f_{u p p e r-b o u n d}^{k}(t)$ is the function defining the upper bound. The weight factors $w_{1}^{k}$ and $w_{2}^{k}$ normalize $D(t)$ and ensure that it equals 1 at the lower and the upper bound.

\subsubsection{Discomfort Reduction}

The reduction in discomfort that an agent experiences as a consequence of executing an activity at a specific location is defined as:

$$
D R\left(t_{e s}, t_{e e}\right)=D\left(t_{e s}\right)-D\left(t_{e e}\right)
$$

where $D\left(t_{e s}\right)$ is the discomfort at the start of execution and $D\left(t_{e e}\right)$ is the discomfort at the end of execution. Using this index introduces a preference for activity/location pairs that yield higher levels of discomfort reduction. Discomfort reduction is a measure that considers agents' past performance.

\subsubsection{Execution Effectiveness}

The execution effectiveness an agent experiences for executing an activity at a given location is defined as:

$$
E E\left(t_{e s}, t_{e e}\right)=\frac{\int_{t_{e s}}^{t_{e e}} f_{e f f e c t}(t) \mathrm{d} t}{t_{e e}-t_{e s}}
$$

where $f_{e f f e c t}(t)$ represents the effectiveness function, $t_{e s}$ is the start of execution, and $t_{e e}$ is the end of execution. Using this index introduces a preference for executing activity/location pairs during efficient time windows (whereby efficiency is defined by whatever the effectiveness function represents, e.g., a combination of several effects like social and cultural norms), and it helps to prevent agents from executing activities during time windows when the agent cannot or can only partially execute the activity (e.g., because the shop closes). Execution effectiveness is a measure that considers agents' immediate and upcoming possibilities.

\subsubsection{Look-Ahead Index}

Atkinson (1994) and Ioannou et al. (2001) highlighted the importance of information about future execution options for scheduling problems with time-window constraints. Effectiveness functions provide information about future execution options. For instance, agents can check shop opening hours to get information on whether they can execute a shopping activity at a given time and for how long, or how long they must wait until the next opportunity to shop will arise. Agents can use such information to plan 
ahead, for instance by postponing activities with more execution options/higher prospective effectiveness in favor of more pressing activities for immediate execution (e.g., do daily shopping duties because shops are going to close soon, therefore postponing some other activities).

The aim of the look-ahead index is to provide agents with an awareness of potentially decreasing activity execution options. A simple approach for the example of the shop opening hours would be to measure the percentage of total time available for a potential execution of shopping in the near future (e.g., within the next four days). This approach has the disadvantage that it does not distinguish between execution options that will open soon and those that will open later. Applying higher weights to execution options that open sooner alleviates this problem. This is done through a convolution of the effectiveness function with an exponential kernel (see Fig. 2), similar to the convolution used to calculate state values. The look-ahead value an agent achieves for executing an activity at time $t$ is defined as:

$$
L A(t)= \begin{cases}1+w_{1} \cdot\left(1-\int_{0}^{h}\left(f_{e f f e c t}(t+x) \cdot \text { kernel }(x)\right) \mathrm{d} x\right) & \text { if } f_{\text {effect }}(t)>0 \\ 1 & \text { otherwise }\end{cases}
$$

where $w_{1}$ is a weight factor, $b$ is the look-ahead horizon of the kernel, and $f_{e f f e c t}(t)$ is the effectiveness function. Intuitively, one could understand $L A(t)$ as an index describing the urgency to execute an activity when prospective execution options decrease. Therefore, it was designed in such a way that it yields a value in the range of $\left[1 . . w_{1}\right]$ ( 1 for low urgency and a value approaching $w_{1}$ for decreasing execution options). Since the heuristic function incorporates $L A(t)$ as a factor, it is important to define it $\geq 1$. For the same reason, it is set to 1 if $f_{\text {effect }}(t) \leq 0$, resulting in an indifference for this index when the effectiveness function prevents an execution. The look-ahead index is a measure that considers agents' prospective opportunities.

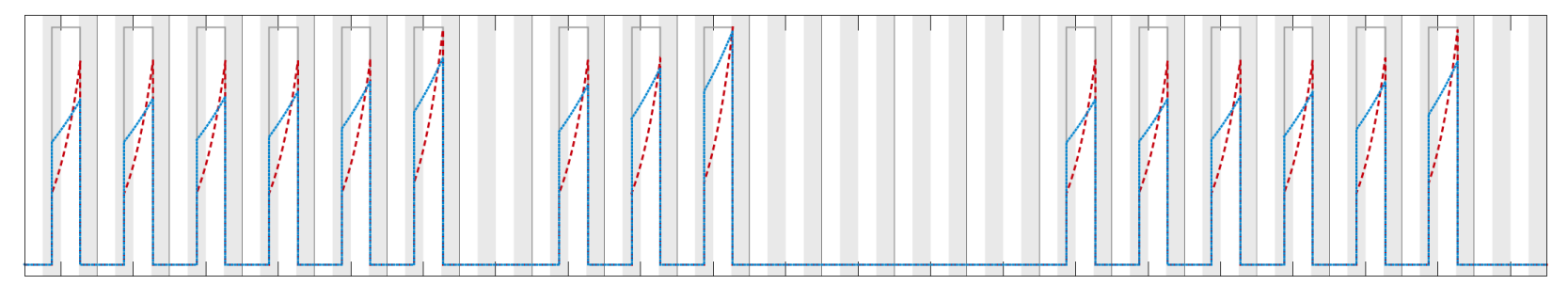

Mon Tue Wed Thu Fri Sat Sun Mon Tue Wed Thu Fri Sat Sun Mon Tue Wed Thu Fri Sat Sun

shop opening pattern

look-ahead (kernel of 7 days)

look-ahead (kernel of 2 days)

Figure 2: Illustration of the look-ahead index for shop opening hours on short and long weekends, comparing different look-ahead windows ( 2 and 7 days). The higher the value, the closer the end of the given shop opening hours window. The value is higher before weekends, indicating fewer shopping options in the near future. The look-ahead index with a kernel of 7 days can differentiate between short and long weekends (value is higher before a long weekend). Choosing the right kernel length is therefore important, so we propose a duration of approximately two to three times the average interval between the execution of two activities of the same type (e.g., $3 \cdot 2$ days $=6$ days for daily shopping). 


\subsubsection{Execution Time Ratio}

The execution time ratio an agent accomplishes for executing an activity at a location is defined as:

$$
\operatorname{ETR}\left(t_{t s}, t_{e s}, t_{e e}\right)=\frac{t_{e e}-t_{e s}}{t_{e e}-t_{t s}}
$$

where $t_{e e}-t_{e s}$ defines the activity duration and $t_{e e}-t_{t s}$ the duration between the start of travel (defined by the end of the last activity at the previous location) and the end of execution $t_{e e}$. Accordingly, a higher share spent traveling results in a smaller factor. This parameter introduces an aversion to traveling and a preference for activity execution. Consequently, it produces a preference for accessible locations (locations that can be reached fast) and fosters activity chaining. The execution time ratio is a measure that considers agents' immediate possibilities as well as predictions of prospective travel times.

\subsubsection{Individual Perception}

A decision procedure that only uses the above components would make homogeneous decisions, i.e., agents having the same state and facing the same situation would make the same decisions. Clearly, this does not match the heterogeneity of observed behavior (see, e.g., Chikaraishi et al. 2010; Schönfelder 2006). A possible workaround to improve heterogeneity is to introduce an unexplained preference, modeled as a random variable altering the perception of alternatives (see, e.g., Horni et al. 2012; McFadden and Train 2000, for other studies modeling heterogeneity in a population by random variables). Agents can incorporate an optional scaled random term into their decision procedure. This step activates agents' individual perceptions and the decision procedure becomes nondeterministic.

\section{Continuous Location Choice}

The aim of this section is to discuss the location choice integrated into the continuous simulation model. The continuous model aims at time horizons that go beyond the 24-hours scenario of other agent-based software packages (e.g., Balmer 2007). As a consequence, a continuous location choice procedure also needs to consider aspects that are in addition to the aspects considered by existing procedures (see, e.g., Horni et al. (2012) for a study concerning MATSim). For instance, it should also consider changing conditions of long-term phenomena (like seasonal effects), mid-term aspects (like recurrent visits of relatives or changing weather conditions), and short-term elements (like expected travel times). The continuous simulation considers these elements by using a location choice procedure that combines expected travel time, current location effectiveness, prospective location effectiveness, and an individual unexplained location preference. The combination of these elements not only allows investigations of aspects with different time horizons (like seasonal aspects and weather conditions) but also enables variety seeking and produces location choice decisions in acceptable time and with a reasonable memory footprint.

The next subsection explains how the decision procedure implements a preference for accessible locations. This is followed by a subsection illustrating how the decision procedure considers changing conditions of aspects with different time horizons. The last subsection presents agents' individual location perception, enabling a nondeterministic location choice procedure.

\subsection{Preference for Accessibility}

People tend to execute their activities (e.g., satisfy their daily shopping needs) in their surroundings. The decision heuristic considers this preference through the execution time ratio (see Eq. (8)). The resulting factor penalizes travel time by being higher for alternatives with a lower travel share. This introduces a preference for accessible locations and activity chaining.

\subsection{Location Effectiveness}

C-TAP aims at the continuous simulation of long time periods like weeks or months. Accordingly, a continuous location choice procedure needs to be able to consider changing conditions of aspects with different time horizons (e.g., long-term phenomena like seasonal effects as well as mid-term aspects like changing weather conditions). C-TAP provides this ability for each individual 
location through the definition of an effectiveness function, informing agents about the effectiveness of a location with respect to discomfort reduction (also see Section 3.2 for a discussion of effectiveness functions).

Effectiveness functions combine different effects (constraints, norms, long-term phenomena, continuous aspects, etc.), which can be static (e.g., precomputed measures like opening hours) or dynamic (e.g., measures computed on-the-fly coming from a sensor observing the environment). Effectiveness functions are considered by the planning heuristic (see Section 4) through discomfort reduction, execution effectiveness and the look-ahead index and as a consequence, they are an important element of the location choice procedure.

Effectiveness functions can model many location choice aspects. The following list gives an idea of the range of problems they can address:

- Congestion-sensitive location choice: One way to prevent location overuse is by incorporating information on crowdedness into the effectiveness function of locations. This example builds on the assumption that executing an activity at overcrowded locations (e.g., shops) is less efficient and therefore takes longer than at less crowded locations. Information on crowdedness can build on people's experience of how many people visit the location at a given time (in this case the function would be static) or on the number of agents actually visiting the location and the space limitation of the location (in this case the function is computed dynamically). The planning heuristic equips agents with a preference for less crowded locations (because discomfort reduces faster) and it urges agents to execute activities before locations get crowded (through the look-ahead index).

- Weather-sensitive location choice: One way to model weather sensitivity is by incorporating weather forecast information into the effectiveness function of locations hosting weather-sensitive activities. This example builds on the assumption that people have a preference to execute weather-sensitive activities when weather conditions are good. The weather information and their categorization can come from external sources (e.g., from the Swiss Federal Office of Meteorology and Climatology) or modelers can come up with weather information that are in accordance with their scenario and the question they want to investigate. The weather categories are then graded by the modeler and receive an effectiveness measure according their closeness to their best category (e.g., sunny weather receives effectiveness 1 whereas heavy snowfall receives effectiveness 0.1). The planning heuristic prevents agents from executing weather-sensitive activities during snowy days (because discomfort reduces slower) and it urges agents to execute them ahead of bad weather fronts (through the look-ahead index).

- Pattern-sensitive location choice: In some contexts, it might be desirable to produce a location choice behavior following certain patterns (e.g., recurrent visits of social contacts like friends or parents). This can be modeled by incorporating information on the elapsed time since the last visit into the effectiveness function of an activity modeling visits to social contacts. This example builds on the assumption that people have a repetitive visiting pattern. Therefore, the effectiveness function increases (e.g., it approaches 1) as the time since the last visit approaches the desired interval time. Since the planning heuristic provides agents with a preference for effective locations, the probability of an agent visiting a social contact also increases as the time since the last visit approaches the desired interval time. The effectiveness function could be assigned to the activity (modeling the meeting pattern to social contacts in general) or to a specific location (modeling the meeting pattern to specific social contacts like parents).

- Seasonal location choice: One way to model seasonal effects is by incorporating seasonal rhythms into the effectiveness function of locations hosting seasonal activities. For example, a ski resort is more effective during the winter months after a snowfall, whereas the yacht club is more effective during the summer months, when sunny weather and a good breeze are more likely. This function enables agents to follow seasonal rhythms by providing a preference to ski at a ski resort during the winter and a preference to sail at a yacht club during the summer (see Section 6 for such an application).

- Income-sensitive location choice: Visits to some locations might depend on income (e.g., visits to luxurious places/commodities). This can be modeled by adding information on income and price level correlation to the effectiveness function of incomesensitive activities and locations (e.g., for a long-term shopping or dine out activity). This example builds on the assumption that wealthier people can afford luxurious places/commodities that poorer people cannot. This is modeled by a dynamic 
effectiveness function taking income and price level into account, resulting in a high effectiveness when levels match and decreasing effectiveness as the difference increases. Since the planning heuristic takes effectiveness into account, agents prefer locations matching their income level.

- Habitual/explorative location choice: One way to model habitual behavior is by incorporating information on visiting frequencies into the effectiveness function of locations. This example builds on the assumption that people's behavior has habitual elements, resulting in a preference for locations they already know. Accordingly, the effectiveness increases as the number of past visits increases. Explorative behavior (counteracting habitual behavior) can be integrated through a decline function, leading to decreasing effectiveness as the time since the last visit increases.

\subsection{Individual Perception}

Individual perception introduces heterogeneity into the location choice procedure using a random variable altering the perception of an agent for an alternative (see also Section 4.2.6). This introduces an individual unexplained preference for certain locations.

Employing random utility theory, the utility of an alternative is

$$
U_{p i}=V_{p i}+\epsilon_{p i}
$$

where $p$ is the person/agent index and $i$ the index of the alternative. $V$ denotes the systematic part of the utility (identical to every agent), $\epsilon$ the random offset, and $U$ the resulting utility. Horni et al. (2012) used a Gumbel distribution and considered three approaches to calculate $\epsilon_{p i}$ and thus, to improve the heterogeneity of their location choice procedure:

(a) Freezing the applied global sequence of random number generation.

(b) Computing and storing a separate $\epsilon_{p i}$ for every combination of agent $p$ and alternative $i$.

(c) Re-calculating $\epsilon_{p i}$ on-the-fly by using a random seed $s_{p i}=f(p, i)$ that depends on agent $p$ and alternative $i$.

Option (a) turned out to be infeasible because it is difficult to freeze a drawing sequence (e.g., in a computer program that runs in parallel) and (b) exceeded memory resources for large-scale scenarios. This left (c) as the only feasible solution that was adopted for the continuous decision procedure presented here. The scaled random term $\epsilon\left(p, i, t_{e s}\right)$ of the planning heuristic is a linear combination of two weighted random variables. The first random variable uses a seed depending on agent $p$ and alternative $i$, providing each agent with a consistent (but different among each agent) order of preference for alternatives. The second random variable uses a seed depending on execution day, altering the variety-seeking behavior between decisions while preserving deterministic behavior between simulation runs. This is necessary because in contrast to Horni et al. (2012), C-TAP does not iteratively simulate the same day but performs a continuous simulation of longer periods where people might face the same decision at different days without choosing the same alternative.

\section{Location Choice Validation}

This section validates the proposed continuous location choice procedure using a model configuration focusing on seasonal aspects, weather conditions, location accessibility, and individual perception for leisure activities. We start with a description of the model configuration and then discuss simulation results.

\subsection{Model Configuration}

We use a grid of $50 \times 50$ cells. Each cell defines the home location of an agent. Each agent can choose between four locations (Fig. 5 shows their positioning on the grid) for executing leisure activities and, as an alternative, agents can always choose to execute activities at home. The locations for leisure activities are split into two summer locations (e.g., executed at the shore of a lake) and two winter locations (e.g., executed at a ski resort). One of each seasonal location type is susceptible to weather conditions. Opening hours of each leisure location starts at 7 a.m. and ends at 8 p.m. The simulation scenario assumes a year of 12 days, 
with three days for each season (days 1-3 for summer, days 4-6 for autumn, days 7-9 for winter, and days 10-12 for spring). Travel times are calculated based on the Euclidean distance between locations, simplifying identification of effects originating from the preference for accessible locations.

Fig. 3 illustrates the functions used by the simulation to create the effectiveness functions for the leisure locations shown in Fig. 4. Leisure locations either use the summer or winter function of Fig. 3a, dependent on being more effective in summer or winter; might integrate the function of Fig. $3 \mathrm{~b}$ dependent on being susceptible to weather conditions; and use the function of Fig. $3 c$ to integrate opening hours into their effectiveness function. This results in four different effectiveness functions, two for locations being more effective in summer-one with (lake 1 - see Fig. 4a) and one without (lake 2 - see Fig. 4b) susceptibility to weather conditions - and two for locations being more effective in winter-one with (ski 1 - see Fig. 4c) and one without (ski 2 see Fig. 4d) susceptibility to weather conditions.

\subsection{Simulation Results}

We perform three simulation runs, one with deactivated individual perception (no randomness) and two with activated individual perception using a small and a large random share. We only activate the second random variable (see Section 5.3) since agents should have a neutral base attitude towards all four leisure locations. Fig. 5 illustrates the simulation results of days 1, 5, 7, and 11. The first picture of each row shows results with deactivated and the second and third pictures with activated individual perception (the second using a small and the third using a large random share). The home cell of an agent traveling to lake 1 is colored orange, to lake 2 yellow, to ski 1 dark blue, and to ski 2 light blue.

- Fig. 5a compares the simulation results of day 1 . Since it is a sunny summer day, agents prefer locations with highest effectiveness in summer (lake 1 and lake 2). With deactivated individual perception, agents choose the closest and most effective location (first figure). The decision border starts to blur as the variance of individual perception increases (second and third figures). These simulations show that agents choose the most effective location and that the influence of effectiveness decreases as individual perception increases.

- Fig. 5 b compares the simulation results of day 5. Since it is a snowy autumn day, agents prefer locations ski 2 and lake 2 because they are insusceptible to weather conditions and because seasonal effects cancel out at day 5 . With deactivated individual perception, agents choose the closest and most effective location (first figure). The decision border starts to blur as the variance of individual perception increases (second and third figures). These simulations show that agents react to weather conditions and choose locations insusceptible to weather when conditions are bad.

- Fig. 5 c compares the simulation results of day 7. Since it is a sunny winter day, agents prefer locations with highest effectiveness in winter (ski 1 and ski 2). With deactivated individual perception, agents choose the closest and most effective location (first figure). The decision border starts to blur as the variance of individual perception increases (second and third figure). These simulations show that agents follow seasonal rhythms and choose to execute winter activities in winter and summer activities in summer (see Fig. 5 a to compare to summer conditions).

- Fig. 5 d compares the simulation results of day 11 . Since it is a sunny spring day and seasonal effects cancel out, all locations have the same effectiveness. Accordingly, agents choose the closest location (first figure) and the decision borders start to blur as the variance of individual perception increases (second and third figure). These simulations show that agents base their decision on locations' accessibility when other effects cancel out.

In the above simulations, we use the Euclidean distance between locations to calculate travel times (simplifying identification of effects originating from the preference for accessible locations). Accordingly, it is not surprising that increasing individual perception (higher random share) moves the travel time distribution towards longer travel times (see Fig. 6). This shows a possibility to calibrate the simulated travel time and travel distance distributions through altering the parameters of the random term. Horni et al. (2011) observed this effect as well and used it to calibrate their model to fit travel distance distributions of the Swiss National Travel Survey (Swiss Federal Statistical Office (BFS) (2006)). 


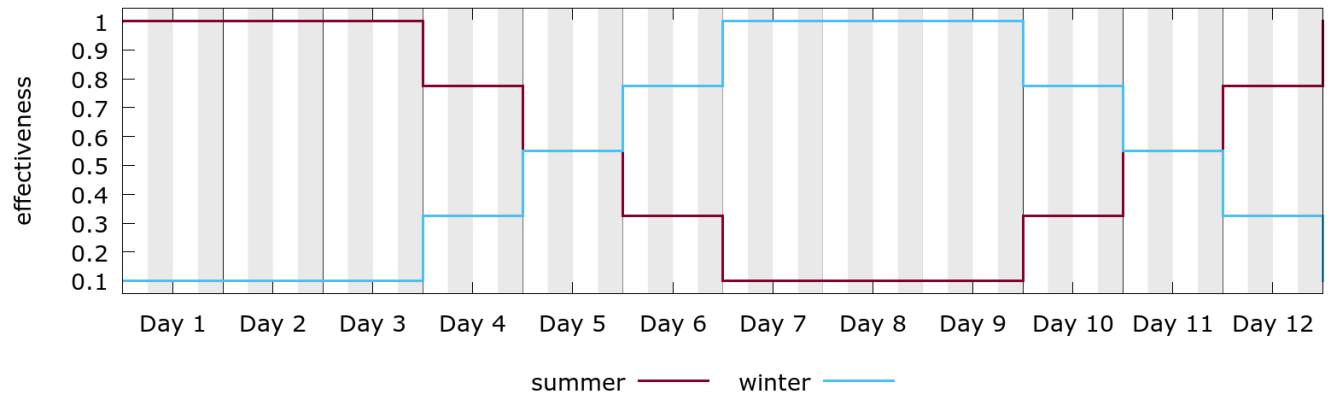

(a) Functions modeling seasonal effects (days 1-3 for summer, days 4-6 for autumn, days 7-9 for winter, and days 10-12 for spring). Locations more effective during summer use the summer function and locations more effective during winter use the winter function.

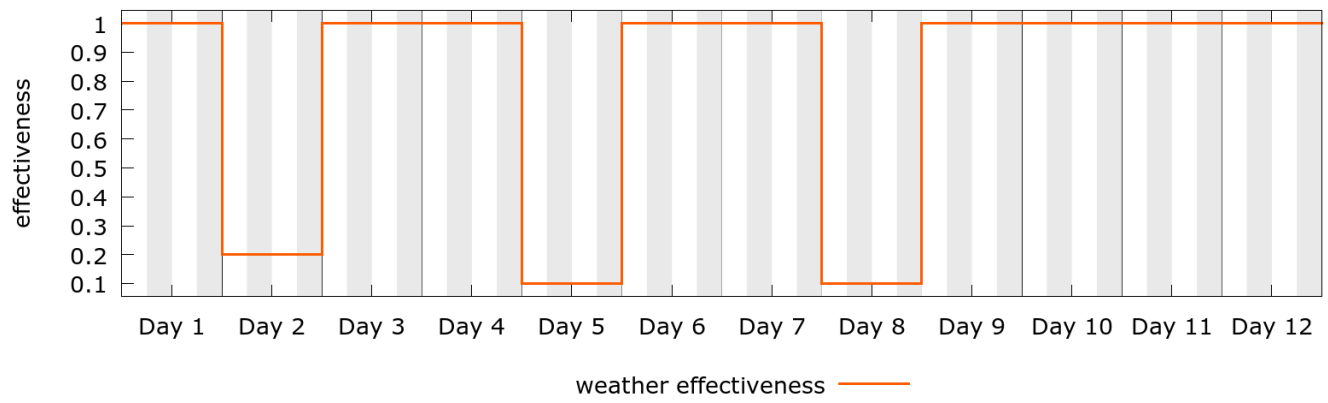

(b) Function modeling weather conditions. Locations integrate this function into their effectiveness if they are susceptible to weather conditions. Day 2 represents a rainy day, days 5 and 8 are snowy days, and all others are sunny days.

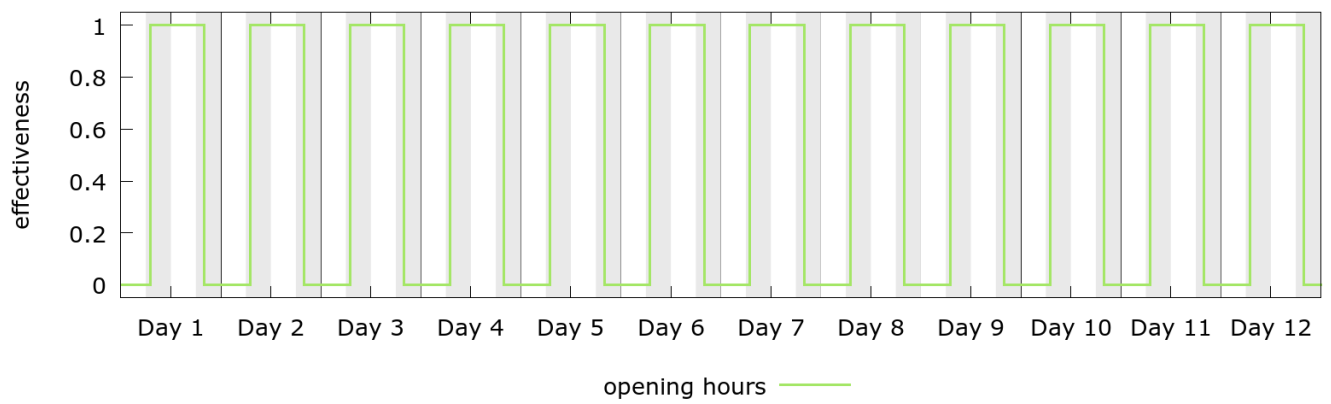

(c) Function modeling opening hours. All locations integrate this function into their effectiveness.

Figure 3: Illustration of the functions used by the simulation to create the effectiveness functions for the leisure locations shown in Fig. 4. 


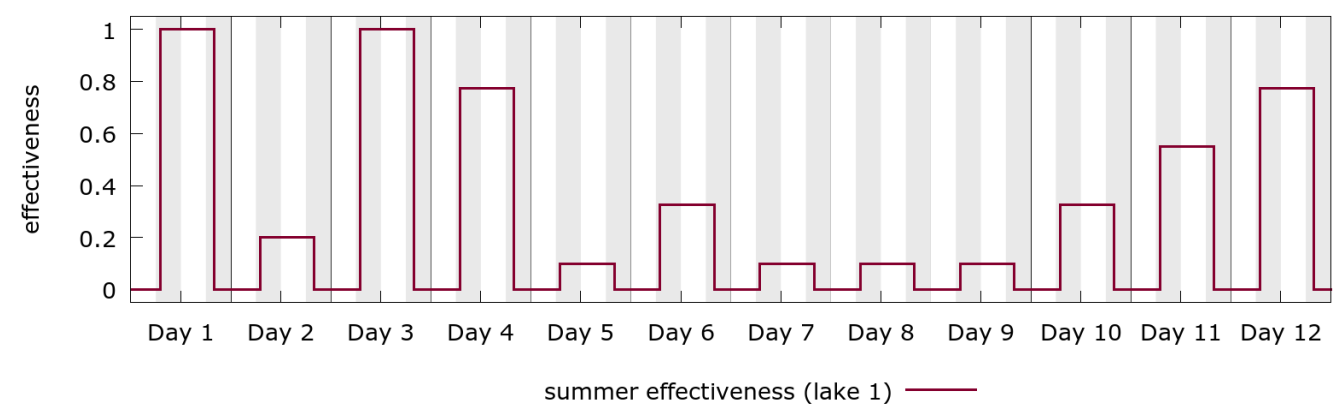

(a) Effectiveness function for the location (lake 1) being more effective in summer. This location is susceptible to weather conditions (days 2, 5, and 8).

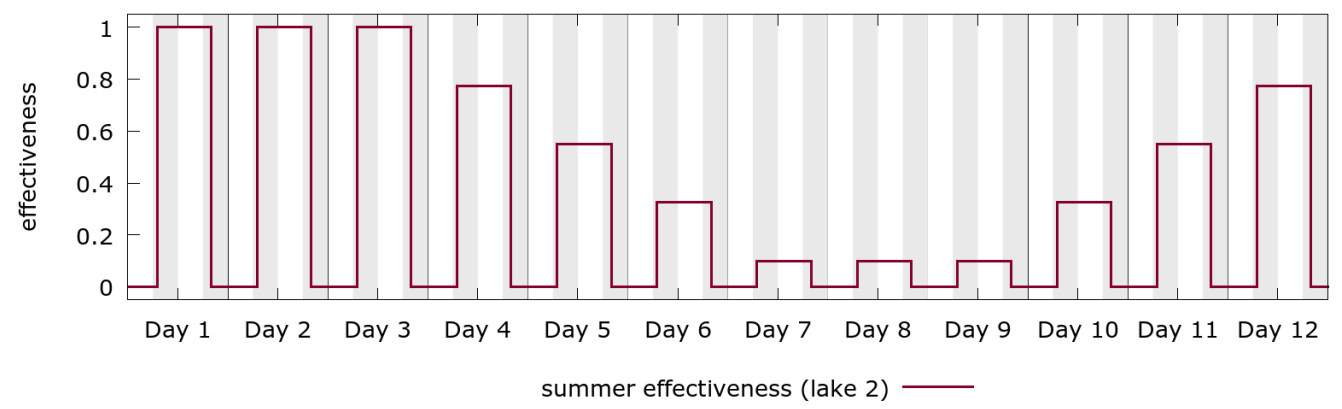

(b) Effectiveness function for the location (lake 2) being more effective in summer. This location is insusceptible to weather conditions.

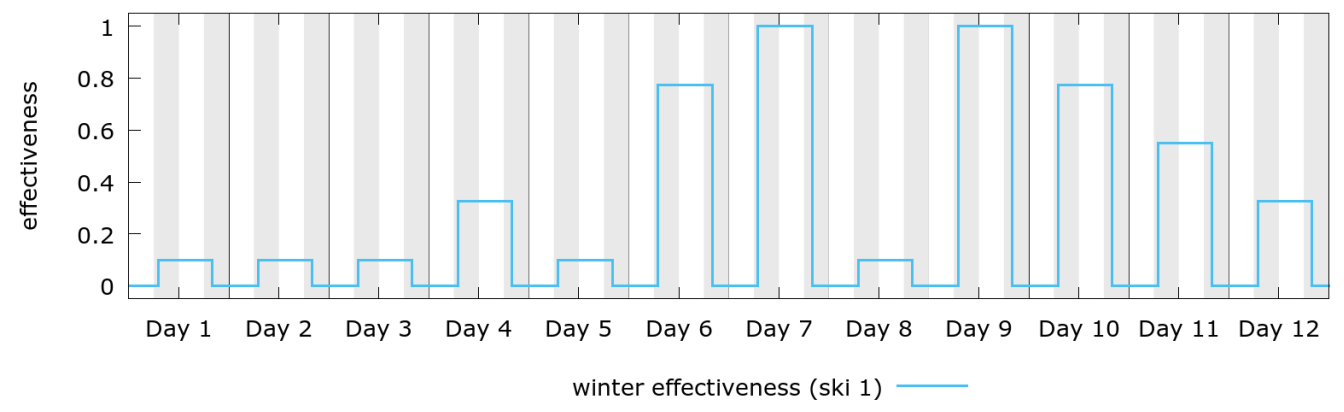

(c) Effectiveness function for the location (ski l) being more effective in winter. This location is susceptible to weather conditions (days 2, 5, and 8).

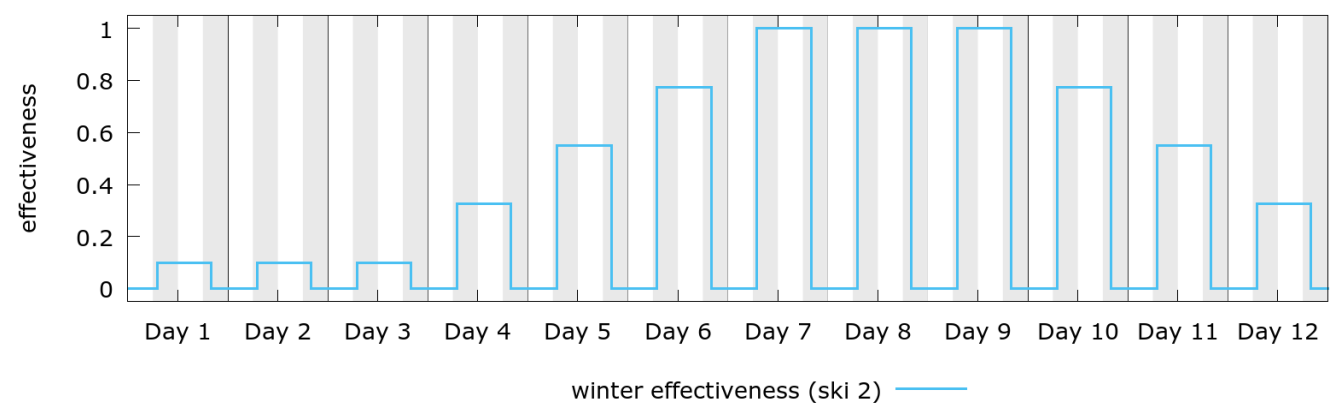

(d) Effectiveness function for the location (ski2) being more effective in winter. This location is insusceptible to weather conditions.

Figure 4: Illustration of the location effectiveness functions created by the simulation using the functions shown in Fig. 3. 

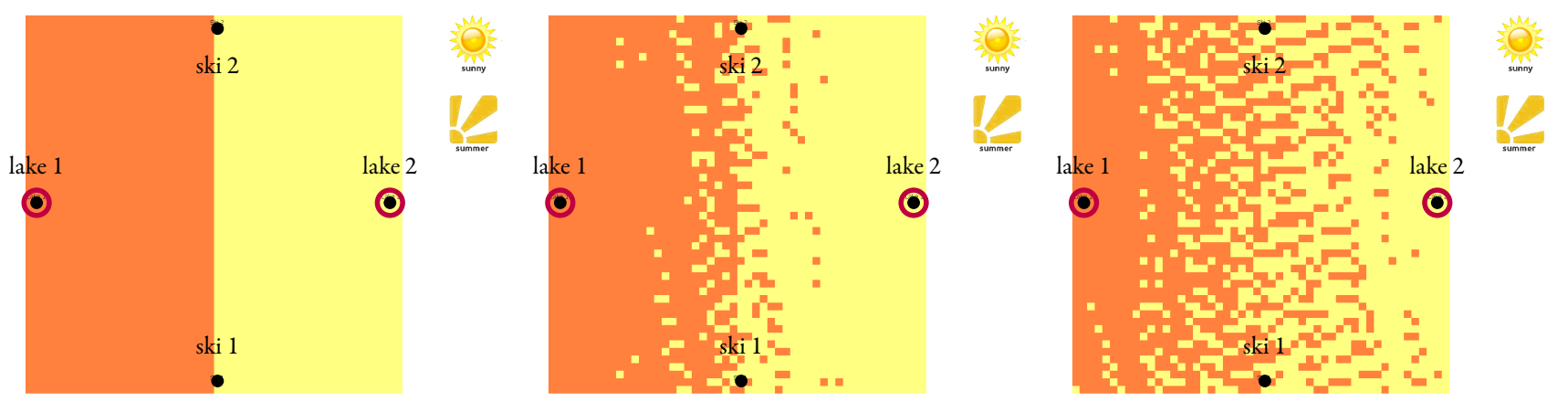

(a) Simulation results of day 1 . A sunny summer day with a preference for summer locations (lake 1 and lake 2).
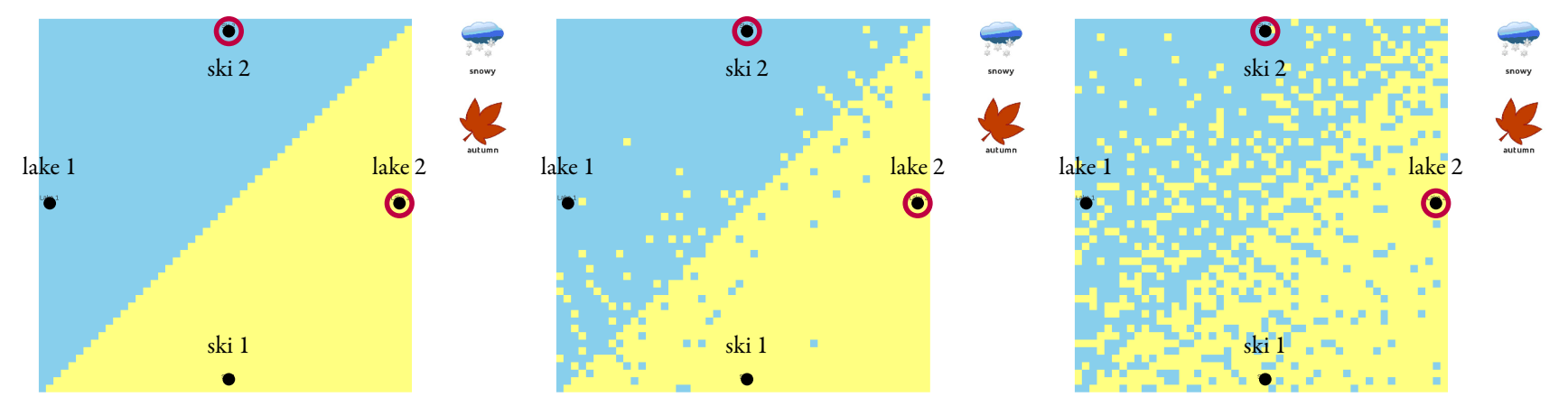

(b) Simulation results of day 5. A snowy autumn day with seasonal effects canceling out and a preference for locations insusceptible to weather conditions (ski 2 and lake 2).
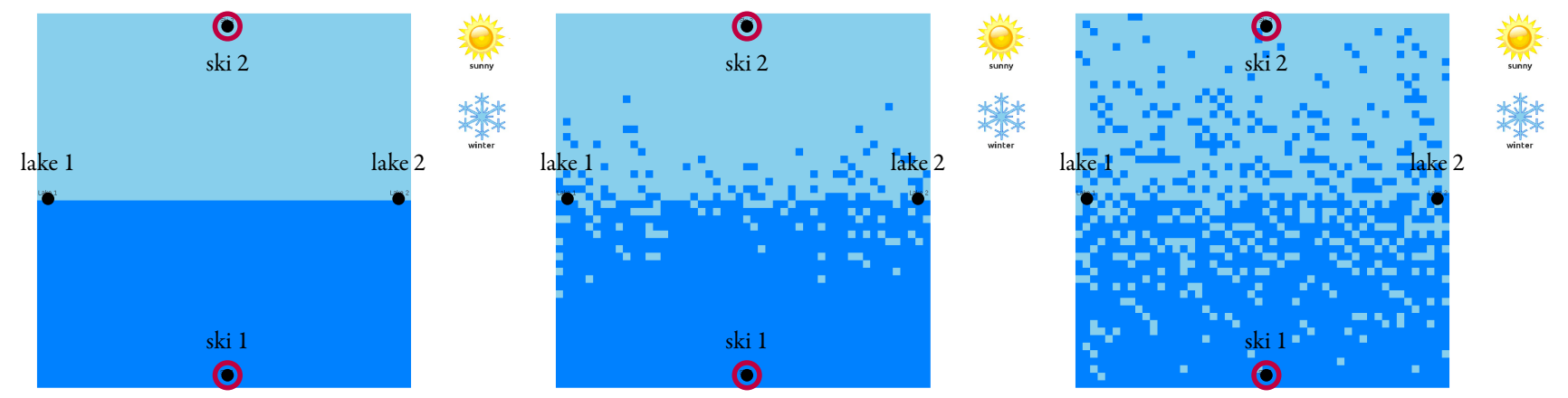

(c) Simulation results of day 7. A sunny winter day with a preference for winter locations (ski 1 and ski 2).
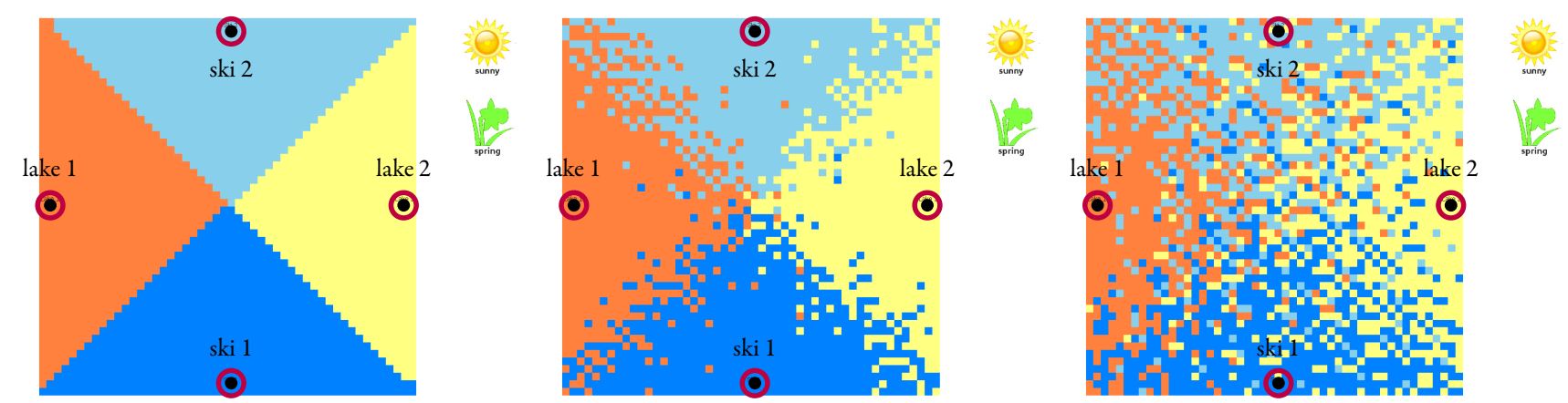

(d) Simulation results of day 11. A sunny spring day with seasonal effects canceling out (agents choose locations based on their accessibility).

Figure 5: Illustration of the simulation results of days 1, 5, 7, and 11 (see Section 6.2 for a detailed discussion). The first picture of each row shows results with deactivated individual perception, the second picture with activated individual perception using a small random share, and the third picture using a large random share. The home cell of an agent traveling to lake 1 is colored orange, to lake 2 yellow, to ski 1 dark blue, and to ski 2 light blue. 


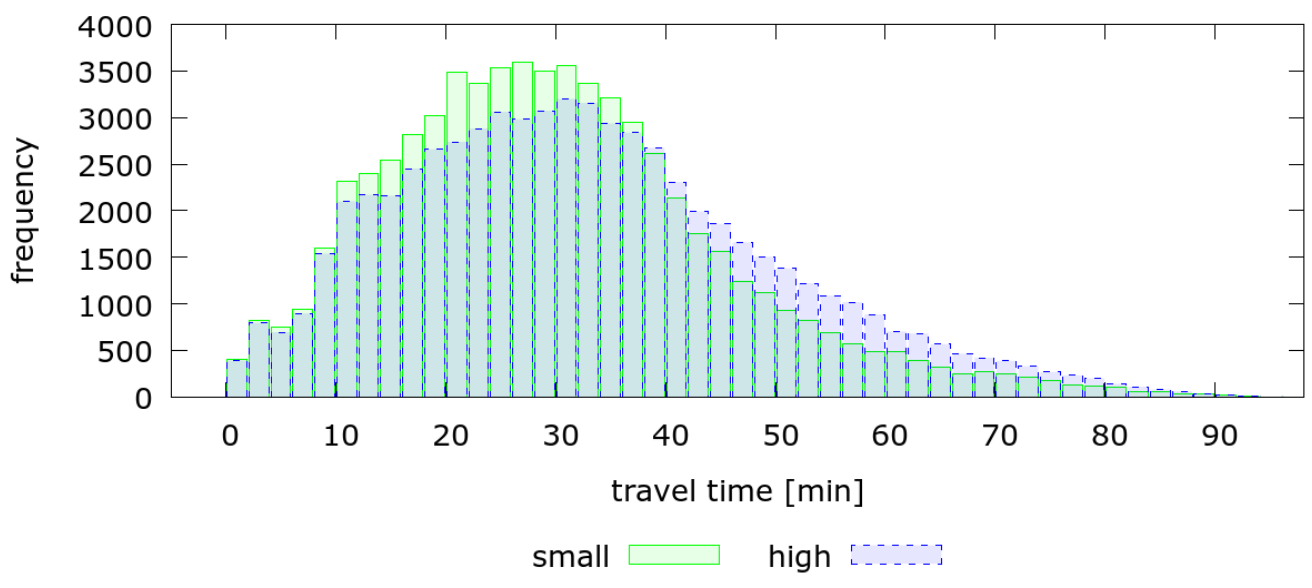

Figure 6: Illustration of the travel time distribution with activated individual perception using a small (green) and a high (blue) random share.

\section{Conclusions}

\subsection{Discussions}

This work is a step towards a travel demand generation framework that allows for a continuous simulation of large scale multi-week scenarios. In the design process, it was important to formulate an extensible approach that uses configuration parameters that have an intuitive interpretation and describe behavior as perceived by people. The proposed approach uses activity execution frequency and percentage of time spent for an activity to characterize targets (modeling agents' internal conditions like goals and preferences) and uses effectiveness functions to describe execution efficacy of activities at locations (modeling external conditions agents encounter at a location). This results in an intuitive model that uses customizable parameters that directly influence behavior, i.e., the effects on the resulting behavior are directly predictable.

Implementing the concept of time horizons by applying a convolution with an exponentially weighted kernel simulates a forgetting process in which agents give more weight to recent behavior and gradually discount their past performance (see Fig. 1b). Similarly, agents also pay more attention to their near future and less to opportunities further in their future (see Fig. 2 for an illustration and Section 4.2.4 for a description). Applying such a decision scheme limits agents' view into their past and future. This has similarities to reality, where people cannot include their complete record and every prospective opportunity in their decision-making process. Consequently, the decision procedure generates decision sequences based on limited information on agents' past performance and future opportunities, similar to a local optimization. Like other authors (e.g., Schlich 2004; Simon 1955), this work takes up the viewpoint that using a nonrecurring decision scheme is behaviorally more authentic than the iterative search for the globally best decision sequences (as e.g., Balmer 2007, applies).

Arentze and Timmermans (2007) pointed out that the dynamics of activity planning range from long-term dynamics related to life trajectories to short-term activity-travel rescheduling behavior, and several time horizons between these extremes. The decision procedure presented here plans activities on-the-fly considering different time horizons. On one hand, this procedure enables agents to react spontaneously to unexpected events since they can continuously adjust their decisions based on a changing environment. On the other hand, the kernel-based approach of targets and look-ahead indices allows agents to consider different time horizons simultaneously. For instance, it would be possible to assign targets with kernel lengths of one week, one month, and one year to a work activity, ensuring that agents adhere to their weekly, monthly, and yearly work quota.

C-TAP derives activity durations from agents' state and external conditions. Agents monitor their performance during the simulation and compare it to their targeted behavior (see Fig. 1b). This allows for varying activity durations because they are based on the time needed to adjust state values to target values (i.e., reduce discomfort). Accordingly, an activity's execution duration varies with the time since its last execution. Furthermore, the execution duration also varies with its execution efficacy defined by the effectiveness function. For instance, if an effectiveness function takes shop crowdedness into account, shopping 
at an overcrowded shop takes longer than at a less crowded shop because the effectiveness influences the increase rate of the state value.

The proposed continuous location choice procedure builds on a combination of expected travel time, current location effectiveness, prospective location effectiveness, and individual unexplained location preference. The combination of these elements not only allows investigations of changing conditions of aspects with different time horizons but also enables variety seeking and produces realistic location choice decisions (see Section 6). Using effectiveness functions as an indication for locations' current and prospective effectiveness with respect to discomfort reduction is a broad concept that can model many aspects (e.g., susceptibility to weather conditions, recurrent visiting patterns, and seasonal effects). Due to the generality of the effectiveness approach, it should be possible to extend the location choice procedure in order to satisfy future modeling needs.

The simulation presented in this work uses OpenMP to parallelize our algorithms, which are implemented in $\mathrm{C}++$. The current algorithms allow for a real-time simulation of approximately one million agents using 16 threads on two Intel Xeon E78837 processors with 8 -cores and $2.66 \mathrm{GHz}$ each. This should render it possible to simulate large-scale scenarios.

\subsection{Future Work}

The long-term goal is to build a scenario for a full one-year simulation. This project should be capable of reproducing annual demand profiles (e.g., Bernard and Axhausen (2008, 2010)) and behavioral rhythms of individuals (e.g., Axhausen et al. (2002); Habib et al. (2008); Schlich and Axhausen (2003)). To the best of our knowledge, Bieger and Lässer (2008) and the Bureau of Transportation Statistics (2012) are the only data sources covering such long periods. Accordingly, we will have to explore possibilities to generate effectiveness functions using other available resources like historical data (e.g., weather conditions or daylight intensities) and options to combine them with information coming from surveys with shorter duration (we are not planning to conduct a new survey). It is also unclear how to recognize and model phase transitions in people's lives (i.e., abrupt changes like the birth of a child) and the time thereafter when this exceptional situation becomes everyday life. We will have to determine to what extent expert knowledge can help to overcome such ambiguities.

\section{Summary}

This paper proposes a continuous location choice procedure and integrates it into C-TAP (Continuous Target-Based Activity Planning) whose decision heuristic considers different planning horizons simultaneously and decides on-the-fly about future location visits. The proposed location choice combines following elements: first, it integrates expected travel time as a preference for accessible locations, i.e., agents prefer close-by locations they can reach fast. Second, it utilizes effectiveness functions as an indication for locations' current and prospective effectiveness with respect to discomfort reduction. Location effectiveness is a broad concept that can model many aspects, e.g., susceptibility to weather conditions, recurrent visiting patterns, and seasonal effects. Third, it uses individual perception to model unexplained preference for certain locations, resulting in an increase in heterogeneity for simulated location choice behavior. The combination of these elements results in a location choice procedure that is capable of handling changing conditions of aspects with different time horizons, enables variety seeking, and produces realistic location choice patterns. Multiple simulation runs validate the continuous location choice procedure showing agents' behavior in various situations.

\section{References}

Arentze, T. A. and H. J. P. Timmermans. 2006. A new theory of dynamic activity generation. In TRB, ed., 85th Annual Meeting of the Transportation Research Board. Washington, D.C.: Transportation Research Board.

Arentze, T. A. and H. J. P. Timmermans. 2007. Modelling dynamics of activity-travel behaviour. In H. K. S. for Transportation Studies, ed., 12th international conference of Hong Kong Society for Transportation Studies. Hong Kong.

Arentze, T. A. and H. J. P. Timmermans. 2009. A need-based model of multi-day, multi-person activity generation. Transportation Research Part B: Methodological, 43(2):251-265. 
Atkinson, J. B. 1994. A greedy look-ahead heuristic for combinatorial optimization: An application to vehicle scheduling with time windows. Journal of the Operational Research Society, 45(6):673-684.

Axhausen, K. W. 1990. Judging the day: A synthesis of the literature on measuring the utility of activity patterns. Working Paper 561, Transport Studies Unit, University of Oxford, Oxford.

Axhausen, K. W., M. Löchl, and R. Schlich. 2007. Fatigue in long duration surveys. Transportation, 34(2):143-160.

Axhausen, K. W., A. Zimmermann, S. Schönfelder, G. Rindsfüser, and T. Haupt. 2002. Observing the rhythms of daily life: A six-week travel diary. Transportation, 29(2):95-124.

Balmer, M. 2007. Travel Demand Modeling for Multi-Agent Traffic Simulations: Algorithms and Systems. Ph.D. thesis, ETH Zurich, Zurich.

Bernard, M. and K. W. Axhausen. 2008. Überprüfung der Schweizerischen Ganglinien.

Bernard, M. and K. W. Axhausen. 2010. Ein neuer Ansatz für standardisierte Ganglinien. Straßenverkehrstechnik, 54(11):689696.

Bieger, T. and C. Lässer. 2008. Travel market Switzerland 2007. Research report, Schweizerische Verkehrswissenschaftliche Gesellschaft (SVWG), St. Gallen. URL http://www.alexandria.unisg.ch/Publikationen/46512.

Bureau of Transportation Statistics. 2012. 1995 American travel survey. webpage. URL http://www.bts.gov/publications/1995_ american_travel_survey.

Charypar, D., A. Horni, and K. W. Axhausen. 2009. Need-based activity planning in an agent-based environment. In IATBR, ed., 12th International Conference on Travel Behaviour Research (IATBR). Jaipur.

Charypar, D. and K. Nagel. 2006. Q-learning for flexible learning of daily activity plans. Transportation Research Record, 1935:163-169.

Chikaraishi, M., J. Zhang, A. Fujiwara, and K. W. Axhausen. 2010. Exploring variation properties of time use behavior based on a multilevel multiple discrete-continuous extreme value model. Transportation Research Record, 2156:101-110.

Dobler, C., M. Kowald, N. Schüssler, and K. W. Axhausen. 2012. Within-day replanning of exceptional events. In TRB, ed., $91 s t$ Annual Meeting of the Transportation Research Board. Washington, D.C.: Transportation Research Board.

Doherty, S. T. 2005. How far in advance are activities planned? Measurement challenges and analysis. Transportation Research Record, 1926:41-49.

Gliebe, J. P. and K. Kim. 2010. Time-dependent utility in activity and travel choice behavior. Transportation Research Record, 2156:9-16.

Habib, K. M. N., E. J. Miller, and K. W. Axhausen. 2008. Weekly rhythm in joint time expenditure to all at-home and out-ofhome activities: Application of Kuhn-Tucker demand system model with multiweek travel diary data. Transportation Research Record, 2054:64-73.

Horni, A., K. Nagel, and K. W. Axhausen. 2011. High-resolution destination choice in agent-based demand models. Working Paper 682, IVT, ETH Zurich, Zurich. URL http://www.ivt.ethz.ch/vpl/publications/reports/ab682.pdf.

Horni, A., K. Nagel, and K. W. Axhausen. 2012. High-resolution destination choice in agent-based demand models. In TRB, ed., 91st Annual Meeting of the Transportation Research Board. Washington, D.C.: Transportation Research Board.

Ioannou, G., M. Kritikos, and G. Prastacos. 2001. A greedy look-ahead heuristic for the vehicle routing problem with time windows. Journal of the Operational Research Society, 52(5):523-537.

Kuhnimhof, T. and C. Gringmuth. 2009. Multiday multiagent model of travel behavior with activity scheduling. Transportation Research Record, 2134:178-185.

Märki, F., D. Charypar, and K. W. Axhausen. 2012a. Target driven activity planning. In TRB, ed., 91st Annual Meeting of the Transportation Research Board. Washington, D.C.: Transportation Research Board.

Märki, F., D. Charypar, and K. W. Axhausen. 2012b. Validation of a continuous simulation model for daily travel. In STRC, ed., 12th Swiss Transport Research Conference. Ascona.

McFadden, D. and K. E. Train. 2000. Mixed MNL models for discrete response. Journal of Applied Econometrics, 15(5):447-470.

Press, W. H., S. A. Teukolsky, W. T. Vetterling, and B. P. Flannery. 2007. Numerical Recipes: The Art of Scientific Computing. Cambridge: Cambridge University Press, 3. edition.

Schlich, R. 2004. Verhaltenshomogene Gruppen in Längsschnitterbebungen. Ph.D. thesis, ETH Zurich, Zurich. 
Schlich, R. and K. W. Axhausen. 2003. Habitual travel behaviour: Evidence from a six-week travel diary. Transportation, 30(1):1336.

Schönfelder, S. 2006. Urban Rhythms: Modelling the Rhythms of Individual Travel Behaviour. Ph.D. thesis, ETH Zurich, Zurich. Simon, H. 1955. A behavioral model of rational choice. Quarterly Journal of Economics, 69(1):99-118.

Swiss Federal Statistical Office (BFS). 2006. Ergebnisse des Mikrozensus 2005 zum Verkehrsverhalten. Swiss Federal Statistical Office (BFS), Neuchatel.

Winston, G. C. 1982. The Timing of Economic Activities. Cambridge: Cambridge University Press. 